\title{
Piezo stimulated currents in marble samples: precursory and concurrent-with-failure signals
}

\author{
I. Stavrakas ${ }^{1}$, C. Anastasiadis ${ }^{1}$, D. Triantis ${ }^{1}$, and F. Vallianatos ${ }^{2}$ \\ ${ }^{1}$ Technological Educational Institution of Athens, Department of Electronics, Athens, 12210, Greece \\ ${ }^{2}$ Technological Educational Institute of Crete, Department of Natural Resources Engineering, Chania, Crete, 73133, Greece
}

Received: 1 July 2002 - Revised: 18 October 2002 - Accepted: 21 October 2002

\begin{abstract}
The Earth's electric field transient variations are promising candidates of earthquake precursors. In order to study the physical mechanisms of such precursory signals, laboratory experiments of uniaxial compression were carried out.

More specifically the behaviour of stressed marble samples from Penteli Mountain was investigated. The samples were subjected to a time-varying uniaxial compression at both variable and constant stress rates. During the first set of experiments weak electric currents were detected during pressure variations. Such Piezo Stimulated Currents (PSC) were detected while stress steps, both positive and negative were applied, the maximum stress never being greater than the elasticity limit. During the second set of experiments stress was applied at a constant rate starting from zero-stress and ending in fracture. In the region beyond the elastic limit a PSC was detected which after reaching a peak suffered a reversal in its polarity just before fracture.

In a third set of experiments the same procedure was applied to previously structurally damaged samples taking care not to fracture them.

In all cases the PSC followed the variation of stress and moreover it was observed that a linear relationship existed between the PSC maxima and the corresponding stress-rate maxima. The mechanism responsible for the described phenomena can be ascribed to the Moving Charged Dislocations model.
\end{abstract}

\section{Introduction}

The need for laboratory experiments concerning rocks under stress emerged in order to study microscopically the possible mechanisms that appear during the preparation of an earthquake event.

The appearance of spontaneous charge production and transient electric and electromagnetic emission (E-EME)

Correspondence to: D. Triantis (ppo@ee.teiath.gr) with the opening and propagation of microcracks has been discussed by several authors in connection to laboratory experiments.(Warwick et al., 1982; Ogawa et al., 1985; Cress et al., 1987; Enomoto and Hashimoto, 1990). In more recent experiments simultaneous E-EME and acoustic emissions (AE) were observed from the opening of microcracks in both piezoelectric and non-piezoelectric materials (Fiffolt et al., 1993; Enomoto et al., 1994; Hadjicontis and Mavromatou 1994, 1996; Chen et al., 1994; Yoshida et al., 1994; Yoshida et al., 1997). In all cases, the intensity of microfracturing and E-EME was accelerated shortly prior to failure and the electric field amplitudes were of the order of $\mathrm{V} / \mathrm{m}$, associated with current densities of the order of $\mathrm{nA} / \mathrm{cm}^{2}$. With the exception of Chen et al. (1994), E-EME was observed only in dry rock specimens.

All the aforementioned experiments were carried out in order to detect experimentally the existence of any electric and electromagnetic effects. In the present contribution the results of measurements concerning the behaviour of marble samples under uniaxial stress are presented. The experimental results demonstrate the appearance of transient electric signals during the stress stage and the failure of a sample. The analysis of the experiments suggests a linear relationship between the emitted current and the stress rate. This linearity is discussed in the frame of the moving charged dislocation model (MCD), (Vallianatos and Tzanis, 1998). Furthermore, for the first time, the dependence of transient electric signals on the application of repetitive loading cycles is presented. The results are discussed introducing the concept of "work hardening" in the MCD model.

\section{Experimental details}

In the present work, Dionysos marbles collected from Mt. Penteli, Attica were used. The Dionysos marble, which has been typically used since the ancient times for the construction of artifacts and monuments, is mainly composed of calcite (98\%) and other minerals depending on the vari- 


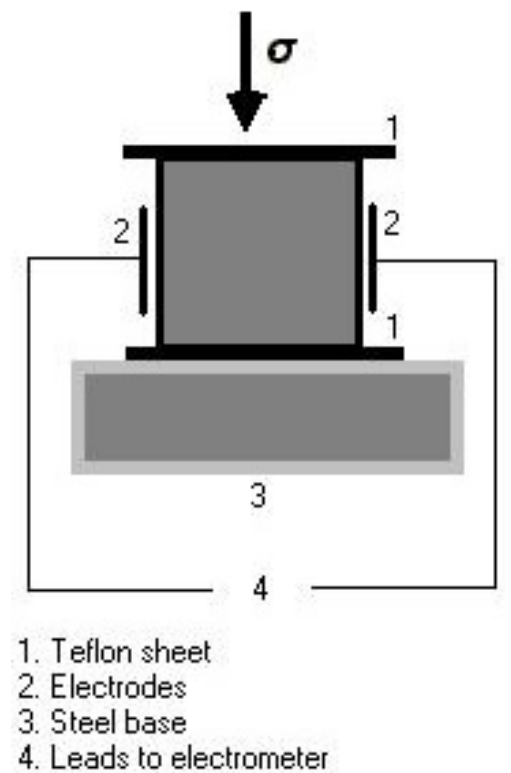

Fig. 1. Placement of the electrodes on the sample for current measurement under uniaxial stress.

ety of the marble, such as muscovite, sericite and chlorite (Kleftakis et al., 2000). Its content in quartz is very low, about $0.2 \%$. Its density is $2.7 \mathrm{~g} / \mathrm{cm}^{3}$ and its porosity is approximately $0.4 \%$. Calcite crystals are polygonic, sometimes exhibiting twinning and mainly of equal size and their texture may be characterized as quasi-homoblastic. The rock is white with a few thin parallel ash-green colored veins containing silver-shaded areas due to the existence of chlorite and muscovite. Matrix rocks were intentionally selected to be single-grained. The experiment has been conducted in a Faraday shield to prevent electric noise from affecting the recorded signals. The noise-protected system comprised a uniaxial hydraulic load machine (EnerpacRC106) that applied compressional stress to the sample, which was placed on a stainless steel base. The marble sample was placed between two thin teflon plates, in the direction of stress, in order to provide electrical isolation. The values of the externally applied compressional stress were recorded using a manometer. A pair of electrodes were attached to the marble sample, using conductive paste. It has to be noted that the electrodes were attached in a direction perpendicular to the axis of the applied stress (see Fig. 1). The measuring system consisted of a sensitive programmable electrometer ( Keithley 617) capable of resolving currents as low as $0.1 \mathrm{fA}$, and as high as $20 \mathrm{~mA}$ in 11 ranges. The data output from the electrometer were stored in a computer using a GPIB interface.

\section{Results}

The technique used to measure the current emitted from rock samples while applying stress at various rates, will henceforth be referred to as the Piezo Stimulated Current (PSC) technique. In the first set of experiments on marble samples,

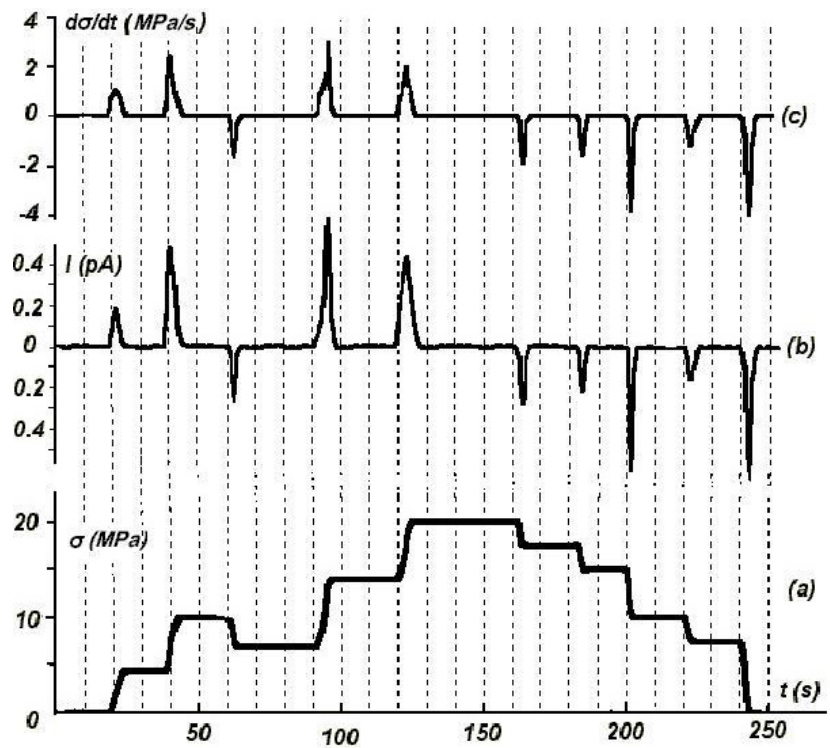

Fig. 2. Correlation of the stress steps (a), current (b) and loading rates (c) with respect to time. The emitted current following the stress steps is shown to be proportional to the rate of change of the applied stress.

abrupt increases and decreases of stress were applied, forming a step-like stress cycle (see Fig. 2a). Figure 2b presents the observed pressure stimulated currents (PSC) during the application of the load cycle.Both positive and negative stress rates $(d \sigma / d t)$ are presented in Fig. 2c. It is clear that there is a correspondence between the curves of PSC and $d \sigma / d t$. Figure 3 presents an attempt to correlate the maximum emitted current $I_{\max }$ at each step and the corresponding maximum stress rate $(d \sigma / d t)_{\max }$. The best straight line fit demonstrates the linearity between the emitted current and the stress rate.

In a second type of experiments, samples extracted from the same matrix rock, were subjected to a constant stress rate, from zero stress to fracture and the emitted piezo-stimulated current was measured. The linear increase of stress with respect to time is depicted in Fig. 4. The selected constant stress rate was $d \sigma / d t=0.3 \mathrm{MPa} / \mathrm{s}$. Figure 4 also depicts the temporal variation of the emitted PSC. The fracture limit of the samples was found to be $51 \mathrm{MPa}$. While the applied stress value was lower than $30 \mathrm{MPa}$ (about $60 \%$ of the failure stress) the released current was not significant. When the stress became greater than $30 \mathrm{MPa}$ a significant current was emitted. At a stress value of $42 \mathrm{MPa}$ (i.e $80 \%$ of the failure stress) a current peak was recorded. At values greater than $42 \mathrm{MPa}$ a steep reduction of PSC was recorded and the polarity of the current was reversed. The negative spike of the measured current, in absolute values, was three times greater than the maximum measured positive current value and was observed systematically just before fracture. It has to be noted that the same behaviour was recorded in all similar experiments that were carried out with all samples of the same matrix rock.

A third set of experiments was conducted to investigate how the samples from the same matrix rock react in the 


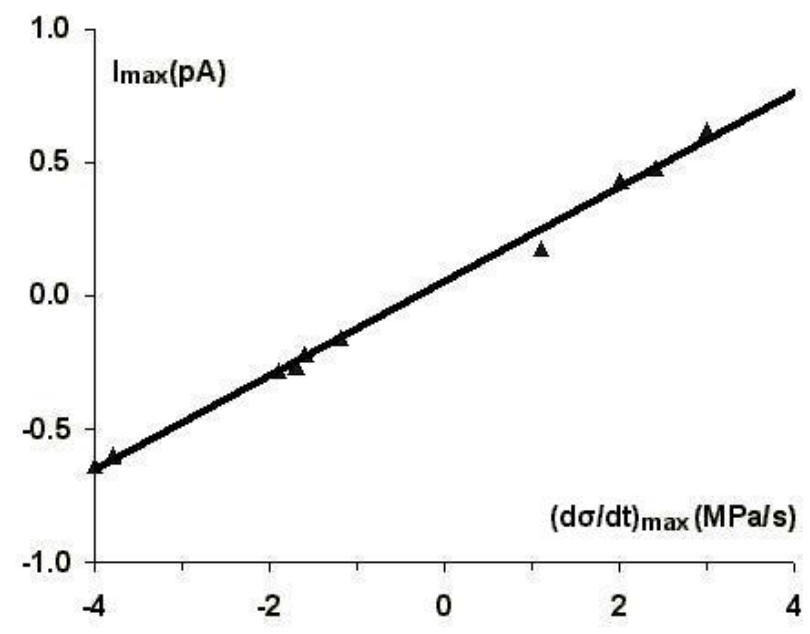

Fig. 3. Correlation of the maximum emitted current during the stress step with the maximum value of stress rate.

case that they had already been damaged by previously applied stress. As previously, the samples were subjected to a constant stress rate of $0.3 \mathrm{MPa} / \mathrm{s}$, from zero to $48 \mathrm{MPa}$, just before failure (Fig. 5, curve a). Significant currents were recorded for stress values over approximately $30 \mathrm{MPa}$. Afterwards the stress was removed and the sample remained unstressed for $30 \mathrm{~min}$. The above procedure was repeated twice. During the second loading cycle, the measured current was smaller than the current observed in the first cycle, as can be seen in Fig. 5 (curve b). This means that the total released electric charge was smaller. During the third repetition of the experiment a very small current was recorded, as can be seen in Fig. 5 (curve c). On increasing the stress above $48 \mathrm{MPa}$, a negative current-spike was recorded as in the previous set of experiments and failure occurred at about $54 \mathrm{MPa}$.

\section{Discussion}

In a crystalline structure, dislocations may form due to the excess or absence of a half-plane of atoms (Vallianatos and Tzanis, 1998,1999b). The edge of this half-plane comprises a dislocation line, around which the physical fields related with it are concentrated. In an ionic structure there will be an excess or absence of a line of ions along the dislocation line, resulting in dislocation charging. We note that the jogs of edge dislocations can be ionically charged. In thermal equilibrium, dislocation lines are electrically neutral (Whitworth, 1975). In dynamic processes when dislocations move, neutrality can no longer be maintained. Let $\Lambda^{+}$be the density of edge dislocations of the type required to accommodate uniaxial compression (or tension) and $\Lambda^{-}$be the density of dislocation of the opposite type. The motion of charged dislocations produces a transverse polarization $P$ (Vallianatos and

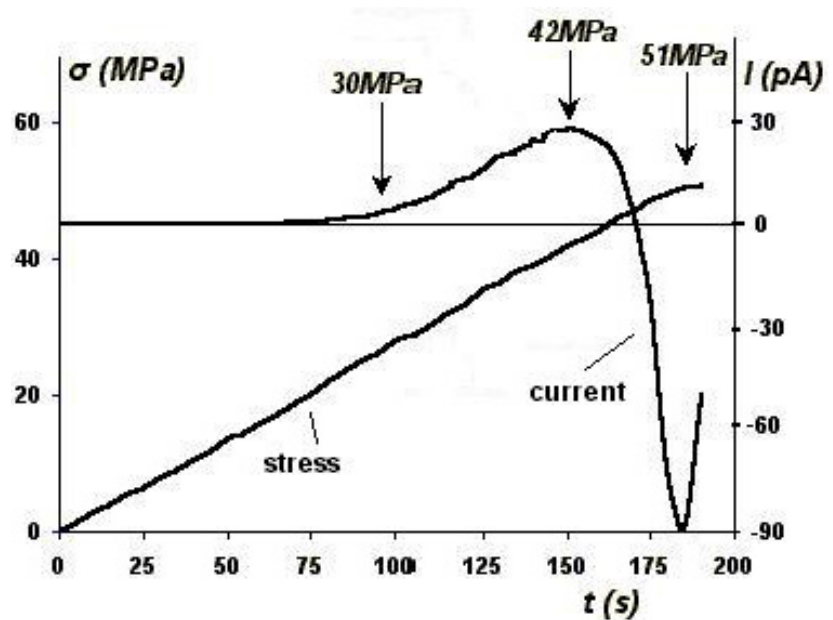

Fig. 4. Correlation of PSC and applied stress with respect to time. On the PSC curve the values of the corresponding stress are noted for the first appearance of PSC, for the maximum PSC and for the fracture.

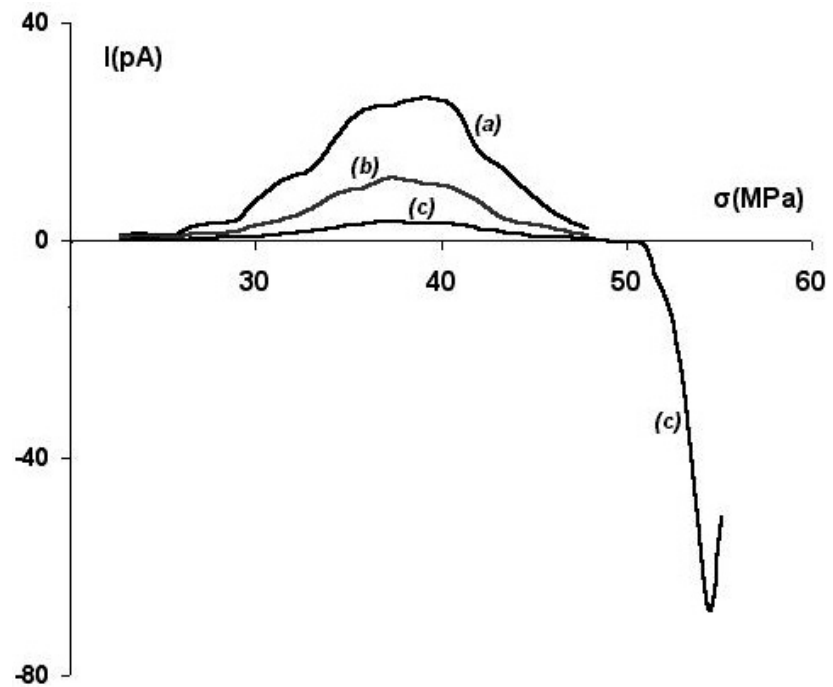

Fig. 5. Consecutive PSC curves at a constant stress rate indicative of the results of the "work hardening" procedure. (a) Initial compression, (b) Second compression, (c) Final compression up to fracture.

Tzanis, 1998) which in turn gives rise to a current:

$I=\frac{\sqrt{2}}{\beta} \cdot \frac{q_{1}}{b} \cdot \frac{A}{Y} \cdot \frac{d \sigma}{d t}=\gamma \cdot \frac{d \sigma}{d t}$,

where $\beta=\frac{\Lambda^{+}+\Lambda^{-}}{\Lambda^{+}-\Lambda^{-}}$, is a constant usually between 1 and 1.5 , $q_{1}$ is the charge per unit length on the dislocation, $b$ is the Burger's vector, $A$ is the electrode area, $Y$ is the Young modulus and $\gamma$ stands for a proportionality factor dependent on the Young modulus of the material. Based on Eq. (1) and taking into account that $Y$ is constant, it can be concluded that: If $\frac{d \sigma}{d t}$ is constant then no current is expected to be recorded $(I=0)$. If $\frac{d \sigma}{d t}>0\left(\frac{d \sigma}{d t}<0\right)$ and $\frac{d^{2} \sigma}{d t^{2}}>0\left(\frac{d^{2} \sigma}{d t^{2}}<0\right)$ then 


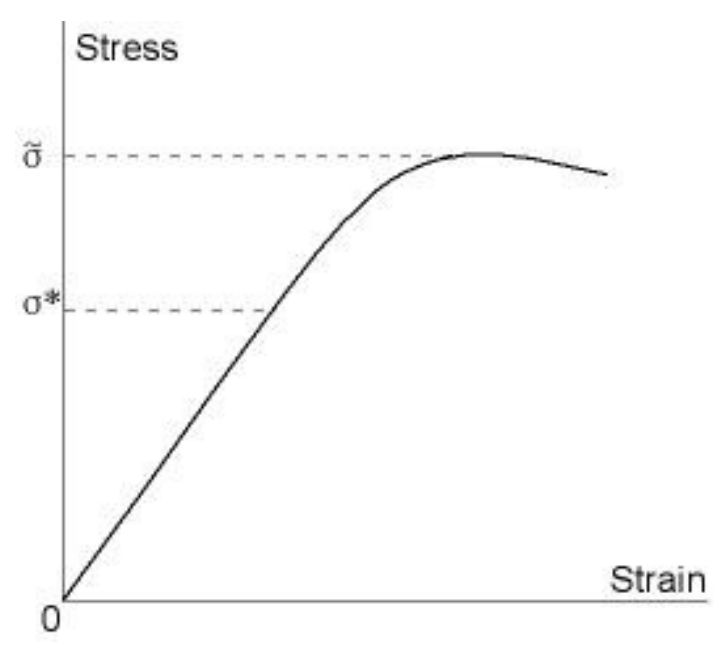

Fig. 6. Typical stress-strain curve for rocks.

an increasing current emission is expected. On the other hand if $\frac{d^{2} \sigma}{d t^{2}}<0\left(\frac{d^{2} \sigma}{d t^{2}}>0\right)$ then a reduction of current emission is expected. Once the Young modulus is involved in Eq. (1) and in order to discuss the results of PSC recordings with respect to the predictions of the above theory, a typical stress-strain curve like the one in Fig. 6 has to be taken into consideration. It is clear that two stress regions have been observed while the applied stress $\sigma$ was increased.

The first region, $\left(\sigma<\sigma^{*}\right)$ where $\sigma^{*}$ denotes the maximum stress in the elastic region, shows a linear relation between stress and current as the strain varies proportionally to the applied stress. When the stress is lower than $\sigma^{*}$, the slope of the stress-strain curve (the Young modulus) is constant and consequently, the factor $\gamma$ in Eq. (1) is constant. Evaluating the results provided by Kleftakis et al. (2000), it seems that this first region continues up to approximately $60 \%$ of the failure stress of the sample.

During the second stage, beyond $\sigma^{*}$, it can be seen that stress and strain are not linearly related. The slope in this region is continuously decreasing. The stress at which the slope equals zero will henceforth be noted by $\tilde{\sigma}$ and represents the ultimate tensile strength. Finally, shortly prior to failure, the slope becomes negative.

Figure 7 roughly represents the changes in the elastic properties of the marble samples during the repetitive stress-strain tests. It should be expected that on removing the previously applied stress, the sample cannot regain its original elastic properties. This is a process known as "work hardening". Another, important observation is that the slope $(d \sigma / d t)$ of the stress-strain curve increases at each loading cycle. According to Eq. (1) a current is emitted when at least one of its factors varies. Thus, in the case of a variable stress rate, a current emission should be detected. Additionally, when the applied stress exceeds the value of $\sigma^{*}$ and the plastic deformation domain is attained, a current emission should be detected due to the change of the Young modulus. At the same

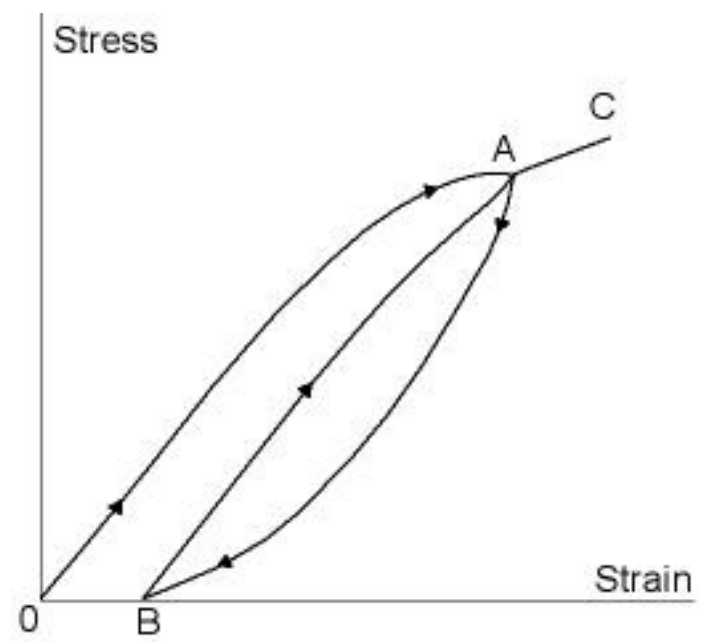

Fig. 7. "Work hardening" model curve in rock samples.

time, when the slope of the stress-strain curve decreases, an increase in the intensity of current emission is expected to take place.

The occurrence of current spikes in the first set of experiments can also be explained by Eq. (1). By varying the quantity $d \sigma / d t$ a corresponding variation in the emitted current is expected. In addition, reversal of polarity of the current spike is expected to follow the change of sign of the stress rate. Equation (1), also explains the observed proportionality between PSC and $d \sigma / d t$ presented in Fig. 3. As is obvious, this experiment was carried out while the applied stress was lower than $\sigma^{*}$ (elastic region), leading to the conclusion that $\gamma$ is constant and that the released current depends only on the quantity $d \sigma / d t$. Finally, it has to be mentioned that when the applied stress was removed, the sample was not plastically deformed.

During the second set of experiments where the stress rate was constant and the sample was led to failure, three distinct regions of corresponding stress-strain variations and PSC emissions were observed. In the first region a weak current was emitted and it is assumed that this was due to the small load (below $\sigma^{*}$, i.e. elastic region) where $Y$ and $d \sigma / d t$ were also constant. The emitted current in this case is expected to be zero. In the second region while $d \sigma / d t$ is constant, an abrupt current emission was observed. This emission can be explained by the decrease of the Young modulus that is taking place while the stress $\sigma$ varies between $\sigma^{*}$ and $\tilde{\sigma}$ and causes $\gamma$ to increase, leading to an increased current intensity. In the third region a negative current spike was observed which corresponds to the final part of the stress-strain curve where the Young modulus becomes negative. The change of the sign of $Y$ implies a corresponding reversal of the polarity of the emitted current.

\section{Concluding remarks}

In the present work the existence of Piezo-Stimulated Currents (PSC) in marble samples is demonstrated. The labora- 
tory results indicate that the PSC is proportional to the stress rate. Furthermore, experiments in order to study in detail the behaviour of rock samples for different applied stresses were carried out. The form of PSC is interpreted taking into account the Moving Charged Dislocation (MCD) model (Vallianatos and Tzanis, 1998, 1999a, 1999b; Tzanis and Vallianatos, 2001, 2002) combined with the general form of the stress-strain curve. Furthermore, the dependence of the emitted current on the order of the loading cycle is demonstrated based on the MCD model and the "work hardening" procedure. The damage of the sample structure due to excessive loading in the plastic region affected greatly the recorded phenomena.

\section{References}

Chen, D., Zhang, D., and Sun, Z.: ULF electric potential changes prior to fracture in rock samples, in: Electromagnetic Phenomena Related to Earthquake Prediction, (Eds) Hayakawa, M. and Fujinawa, Y., Terra Scientific Publishing Co., Tokyo, 323-329, 1994.

Cress, G. O., Brady, B. T., and Rowell, G. A.: Sources of electromagnetic radiation from fracture of rock samples in laboratory, Geophys. Res. Lett., 14, 331-334, 1987.

Enomoto, J. and Hashimoto, H.: Emission of charged particles from indentation fracture of rocks, Nature, 346, 641-643, 1990.

Enomoto, Y., Shimamoto, T., Tsutsumi, A., and Hashimoto, H.: Transient electric signals prior to rock fracturring: Potential use as an immediate earthquake precursor, in: Electromagnetic Phenomena Related to Earthquake Prediction, (Eds) Hayakawa, M. and Fujinawa, Y., Terra Scientific Publishing Co., Tokyo, 253259, 1994.

Fiffolt, D. A., Petrenko, V. F., and Schulson, E. M.: Preliminary study of electromagnetic emissions from cracks in ice, Philosophical Magazine B, 67, 289-299, 1993.

Hadjicontis, V. and Mavromatou, C.: Transient electric signals prior to rock failure under uniaxial compression, Geophys. Res. Lett., 21, 1687-1690, 1994.

Hadjicontis, V. and Mavromatou, C.: Laboratory investigation of electric signals preceding earthquakes, in: A critical review of
VAN, (Ed) Lighthill, Sir J., World Scientific, Singapore, 105117, 1996.

Kleftakis, S., Agioutantis, Z., and Stiakakis, C.: Numerical Simulation of the uniaxial compression test including the specimenplaten interaction, Computational methods for shell and spatial structures, IASS-IACM, 2000.

Ogawa, T., Oike, K., and Miura, T.: Electromagnetic radiation from rocks, J. Geophys. Res., 90, 6245-6249, 1985.

Tzanis, A. and Vallianatos, F.: A critical review of ULF electric earthquake precursors, Annali di Geofisica, 44, 429-460, 2001.

Tzanis, A. and Vallianatos, F.: A physical model of electrical earthquake precursors due to crack propagation and the motion of charged edge dislocations, in: Seismo Electromagnetics: Lithosphere-Atmosphere-Ionosphere Coupling, (Eds) Hayakawa, M. and Molchanov, O. A., TERRAPUB, Tokyo, 117130, 2002.

Vallianatos, F. and Tzanis, A.: Electric current generation associated with the deformation rate of a solid: Preseismic and coseismic signals, Phys. Chem. Earth, 23, 933-938, 1998.

Vallianatos, F. and Tzanis, A.: A model for the generation of precursory electric and magnetic fields associated with the deformation rate of the earthquake focus, in: Atmospheric and Ionospheric electromagnetic phenomena associated with Earthquakes, (Ed) Hayakawa, M., Terra Scientific Publishing Co, Tokyo, 287-305, 1999a.

Vallianatos, F. and Tzanis, A.: On possible scaling laws between electric earthquake precursors (EEP) and earthquake magnitude, Geophys. Res. Lett., 26, 13, 2013-2016, 1999b.

Warwick, J. W., Stoker, C., and Meyer, T. R.: Radio emission associated with rock fracture: Possible application to the great Chilean earthquake of 22 May 1960, J. Geophys. Res., 87, 28512859, 1982.

Whitworth, R. W.: Charged dislocations in ionic crystals, Adv. Phys., 24, 203-304, 1975.

Yoshida, S., Manjgaladze, P., Zilpimiani, D., Ohnaka, M., and Nakatani, M.: Electromagnetic emissions associated with frictional sliding of rock, in: Electromagnetic Phenomena Related to Earthquake Prediction, (Eds) Hayakawa, M. and Fujinawa, Y., Terra Scientific Publishing Co., Tokyo, 307-322, 1994.

Yoshida, S., Uyeshima, M., and Nakatani, M.: Electric potential changes associated with a slip failure of granite: Preseismic and coseismic signals, J. Geophys. Res., 102, 14 883-14 897, 1997. 